\title{
Grupo focal com residentes multiprofissionais no contexto da pandemia COVID-19: Relato de experiência
}

\author{
Nádia Cristina Cardoni' ${ }^{1}$ Mara Quaglio Chirelli' e Danielle Abdel \\ Massih Pio
}

\author{
${ }^{1}$ Faculdade de Medicina de Marília, Brasil | nadia cardoni@hotmail.com; \\ marachirelli@gmail.com; danimassihpio@hotmail.com | https://orcid.org/0000-0003- \\ 1381-9421; https://orcid.org/0000-0002-7417-4439; https://orcid.org/0000-0003-0738- \\ 4601
}

Resumo: Introdução: Em dezembro de 2019, os primeiros casos relatados da ocorrência da SARS-CoV-2, nome da síndrome respiratória ocasionada pelo novo coronavírus, foram inicialmente detectadas em Wuhan, capital da província da China Central. Com comportamento errático e de fácil disseminação fez com que a Organização Mundial de Saúde (OMS), elevasse a doença ao status de Pandemia. Sendo necessário a adoção de uma série de medidas preventivas, como o distanciamento social. $\mathrm{O}$ isolamento social, fez escolas, faculdades e universidades brasileiras interrompesse suas atividades, assim, tornaram-se necessárias estratégias para a manutenção das atividades de ensino e pesquisa, destacando-se a substituição das atividades presencias de ensino por remotas. Neste sentido, todos os processos de investigações científicas nas universidades foram impactados pela pandemia. Portanto, dentro das abordagens qualitativas, as estratégias de coleta de dados, dentre essas o uso do grupo focal (GF), também necessitou de adaptações. Objetivo: O objetivo do estudo foi relatar a estratégia de coleta de dados por meio de GF online no contexto da pandemia pela COVID-19. Metodologia: Trata-se de um estudo descritivo do tipo relato de experiência sobre o uso do GF na coleta de dados, considerando a situação pandêmica pela COVID-19 realizada a partir da investigação de ações desenvolvidas pelos Programas de Residência Integrada Multiprofissional (RIMS) em Saúde Coletiva e Saúde Mental em município da região centro-oeste do estado de São Paulo, Brasil, durante o período de novembro a dezembro de 2020. Resultados: A técnica de GF surge como uma abordagem qualitativa de coleta de dados e uma estratégia de ponte para a pesquisa científica e o conhecimento local. Conclusão: Conclui-se, portanto, que a realização do GF na modalidade online pode sim ser considerada como uma ferramenta alternativa em cenários de pandemia como os vivenciados atualmente, que resultam na impossibilidade ou redução de se realizar reuniões presenciais.

Palavras-chave: Pandemias; Aprendizagem Ativa; Pesquisa Qualitativa; Grupos Focais.

Focus Group with Multiprofessional Residents in the Context of the Pandemic COVID-19: Experience Report

Abstract: Introduction: In December 2019, the first reported cases of the occurrence of SARS-CoV-2, the name of the respiratory syndrome caused by the new coronavírus, were initially detected in Wuhan, capital of the province of Central China. With erratic behavior and easy dissemination, the World Health Organization (WHO) raised the diasease to Pandemic status. It is necessary to adopt a series of preventive measures, such a social detachment. Social isolation, made Brazilian schools, colleges and universities interrupted their activities, thus, strategies became necessary for the maintenance of teaching and research activities, highlighting the substituion of presential teaching activities for remote ones. In this sense, all scientific research processes at universities were impacted by the pandemic. Therefore, within qualitative approaches, data collection strategies, including the use of the focus group (FG), also needed adptations. Goals The objective of the study was to report the data collection strategy through online FG in the context of the pandemic by COVID-19. Methods This is a descriptive study of the type of experience report on the use of FG in data collection, considering the pandemic situation by COVID-19 carried out thought the investigation of actions developed by the Multiprofessional Integrated Residency Programs (RIMS) in Public Health and Mental Health in municipality in the midwest region of the state of São Paulo, Brazil, from November to December 2020. Results The FG technique emerges as a qualitative approach to data collection and a bridge strategy for scientific research and local Knowledge. Conclusion It concluded, therefore, that the realization of the FG in the online modality can indeed be considered as alternative tool in pandemic scenarios such as those currently experienced, which result in the impossibility or reduction of holding face-to-face meeting.

Keywords: Pandemics; Active Learning; Qualitative Research; Focus Groups. 


\section{Introdução}

Em dezembro de 2019, as autoridades sanitárias chinesas informaram à Organização Mundial da Saúde (OMS) a ocorrência de casos de síndrome respiratória aguda grave, com etiologia microbiana desconhecida, na cidade de Wuham, capital da província da China Central. Poucos dias depois, um novo coronavírus foi detectado e a nova doença recebeu o nome oficial de COVID-19 (Souto \& Travassos, 2020); (Hammerschmidt, Bonatelli, \& Carvalho, 2020).

Com comportamento errático e de fácil disseminação e com o aumento do número de casos, rapidamente caracterizou a infecção como um surto, de modo que, no início de março fez com que a OMS, elevasse a doença ao status de Pandemia (Oliveira, Lucas, \& Iquiapaza, 2020).

No Brasil, o primeiro caso foi confirmado em 26 de fevereiro de 2020, e a primeira morte em 17 de março do mesmo ano (Barbosa Júnior et al., 2020). Em 20 de março, o país reconheceu a ocorrência de transmissão comunitária da doença, e a partir de então, medidas de isolamento social para toda população foram recomendadas pelo Ministério da Saúde (MS) (Lucena et al., 2020).

Os sintomas clínicos mais comuns são febre alta, mal-estar, tosse e dificuldades respiratórias, a afetação bilateral dos pulmões é o resultado mais encontrado nas imagens de tomografia computadorizada nos pacientes infectados. (Schmidt, Crepaldi, Bolze, Neiva-Silva, \& Demenech, 2020).

Em função disso, países então adotando uma série de medidas preventivas, por exemplo, o distanciamento social, uso de Equipamentos de Proteção Individual (EPI) a limitação ou interrupção de voos internacionais, a fim de conter a disseminação do vírus (Barbosa Júnior et al., 2020). E, mais recentemente o uso da vacina em caráter emergencial (Quintella, da Mata, Ghesti, \& Tavares, 2020).

Neste sentido, o uso do isolamento social, como forma de atenuar a disseminação e evitar o colapso dos sistemas, modificou vários setores (Barbosa Júnior et al., 2020), fez escolas, faculdades e universidades brasileiras interrompesse suas atividades, assim, tornaram-se necessárias estratégias para a manutenção das atividades de ensino e pesquisa, destacando-se a substituição das atividades presencias de ensino pelo uso das Tecnologias da Informação e Comunicação (TICs) (Barbosa Júnior et al., 2020); (Souto \& Travassos, 2020); (Hammerschmidt et al., 2020). As atividades para alguns segmentos, como o da pós-graduação modalidade formação em residência multiprofissional em saúde, se mantiveram presenciais, uma vez que puderam permanecer nos cenários de prática profissional tendo os devidos cuidados de acordo com os protocolos recomendados, tendo mudanças de locais e tipos de atividades ao longo do período de pandemia.

Sendo assim, todos os processos de investigações científicas nas universidades foram impactados pela pandemia, no que diz respeito as pesquisas qualitativas, esse processo de transformação também ocorreu. A pesquisa qualitativa que tem como objetivo responder a questões muito particulares, dessa forma, ela trabalha com o universo de significados, motivos, aspirações, crenças, valores e atitudes, o que corresponde a um espaço mais profundo das relações utilizando como matéria a experiência humana (Minayo, Deslandes, \& Gomes, 2011).

Os pesquisadores também tiveram que reconstruir suas estratégias de investigação, uma vez que a situação de pandemia exige ações de proteção com relação ao vírus com distanciamento social e medidas de biossegurança. Portanto, dentro das abordagens qualitativas, as estratégias de coleta de dados, dentre essas o uso do grupo focal (GF), também necessitou de adaptações para que pudesse ser desenvolvido. A partir da experiência vivenciada os pesquisadores questionam se a forma de coleta de dados por meio do GF online poderia ou não ter interferido ou contribuído na investigação em curso. Dessa forma, foi proposta a reflexão a partir do relato da experiência para captar os possíveis desafios e potencialidades, podendo contribuir com novas estratégias de coleta de dados. 


\subsection{O Grupo Focal como Estratégia de Coleta de Dados na Pesquisa Qualitativa}

O GF é considerado uma técnica de coleta de dados especifico das pesquisas qualitativas, por proporcionar a interação entre os participantes e o pesquisador para a produção de dados, a partir de discussões focadas em tópicos norteadores (Busanello et al., 2013). Uma pesquisa que utiliza o GF busca desenvolver a compreensão do grupo participante, do seu próprio ponto de vista. Geralmente, o grupo é composto por quatro a oito participantes, o pesquisador e observador. Desta forma, os participantes são selecionados por apresentarem algumas caraterísticas em comum que estão associados ao contexto que está sendo pesquisado (Busanello et al., 2013).

As entrevistas têm duração em média de uma hora e meia. A coleta de dados por GF tem como uma de suas maiores riquezas basear-se na tendência humana de formar opiniões e atitudes na interação com outros indivíduos e está técnica vem sendo aplicado em estudos diagnósticos de problemas educativos em saúde, e em estudos avaliativos de programas de saúde (Ressel et al., 2008).

Tendo em vista que a técnica por meio de grupo focal, por suas próprias caraterísticas, deve ser realizada por composição de grupos modestos, as discussões são grupais, devendo ser fomentadas por uma série de perguntas abertas, estimulantes para explorar a temática e ocorrer em um ambiente agradável e confortável para os participantes.

\section{Objetivo}

Relatar a estratégia de coleta de dados por meio de grupo focal online no contexto da pandemia pela COVID-19.

\section{Metodologia}

Trata-se de um estudo descritivo do tipo relato de experiência sobre o uso do grupo focal na coleta de dados, considerando a situação pandêmica pela COVID-19.

Foi conduzida uma pesquisa qualitativa, do tipo exploratória, realizada a partir da investigação de ações desenvolvidas pelos Programas de Residência Integrada Multiprofissional (RIMS) em Saúde Coletiva e Saúde Mental em município da região centro-oeste do estado de São Paulo, Brasil, durante o período de novembro a dezembro de 2020.

De forma geral, os programas de residências estão envolvidos na proposta de uma formação de qualidade, comprometidos com a mudança em se produzir saúde, com a superação da dicotomia teoria e prática e a capacidade da formação em serviço possibilitar, por meio da presença das universidades nas unidades de saúde, a transformação dos serviços (Mueller, 2018). Portanto, deve se priorizar o crescimento profissional e institucional, envolvendo técnicos e gestores na atenção, na formação e no controle social (Rocha \& Lucena, 2018); (Mueller, 2018).

Neste contexto, o relato de caso deste estudo foi extraído do cotidiano profissional dos residentes mediante suas experiências adquirida durante a pandemia da COVID-19 bem como suas vivências associadas com os desafios e as incertezas neste momento. Os dados aqui relatados traduzem a vivência presencial, observações e discussões entre os residentes nos mais diversos cenários na atenção primária e secundária onde são desenvolvidas suas atividades de trabalho (Rodrigues \& da Silva, 2020), na perspectiva de encontrar novas ideias, para possibilitar um progresso intelectual e mostrar novos problemas e soluções (Kienle \& Kiene, 2011).

Portanto, será apresentada a descrição de como utilizou-se a técnica do GF nessa situação de investigação e, posteriormente, será construída reflexão sobre a experiência. 


\subsection{Relato de Experiência}

Os dados da pesquisa foram obtidos por intermédio da técnica do GF online, por meio de plataforma digital Google Meet. Portanto, as entrevistas online tiveram duração em média de uma hora e meia, e a dinâmica grupal, foi conduzida por um pesquisador que desenvolveu as atividades com roteiro de questões disparadoras e por outro pesquisador que realizou a observação.

Para a seleção e organização do GF online, foi imprescindível ter claro os critérios de inclusão dos participantes na pesquisa, sendo esses: ser residente que ingressou nos anos de 2019 e 2020 nos dois programas de RIMS Saúde Coletiva e Saúde Mental, ou seja, residentes do $2^{\circ}$ ano $(R 2)$ e residentes do $1^{\circ}$ ano $(R 1)$, respectivamente, e ter pelo menos seis meses de vivência na metodologia de ensino-aprendizagem e trabalho. $\mathrm{O}$ critério de exclusão foi estar em afastamento das suas atividades por motivos médicos ou férias.

Todos os residentes R1 e R2 da Saúde Coletiva e Saúde Mental foram convidados a participar da pesquisa, sendo separados em três grupos, dois grupos de R1 composto por seis residentes da RIMS Saúde Coletiva (G1) e cinco da RIMS Saúde Mental (G2), ingressos no ano de 2020 e outro grupo composto por R2 em um total de três residentes da RIMS Saúde Coletiva e três da Saúde Mental (G3) ingressos em 2019.

A pesquisa foi aprovada por Comitê de Ética em Pesquisa da instituição de ensino, sendo aprovada pelo parecer n.4.386.937/2020, tendo como objetivo geral analisar as dimensões do cuidado e da formação na atuação dos residentes no contexto dos Programas de Residência Multiprofissional de Marília/SP, na perspectiva de residentes, preceptores e tutores, durante a pandemia da COVID-19 na atenção primária e secundária a saúde.

Inicialmente, o pesquisador fez contato com os coordenadores dos Programas de RIMS, explicando o objetivo da pesquisa e solicitando o contato dos participantes da pesquisa. A partir dos contatos, fez convite para os residentes R1 e R2 utilizando aplicativo de mensagens para pactuar datas e horários em comum a todos. Foi enviada também aos residentes uma carta-convite a qual constava os esclarecimentos sobre os objetivos, duração dos encontros e desenvolvimento da pesquisa. Além do Termo de Consentimento Livre e Esclarecido (TCLE) online pelo Google Forms que após confirmação por parte dos participantes, era agendado o período e horário do encontro para a coleta de dados.

Realizou-se teste piloto para análise e verificação de necessidades de ajustes no instrumento de coleta de dados com os residentes de outro programa, RIMS da clínicocirúrgica $\mathrm{R} 1$, que não participaria da investigação, mas que também tem vivenciado o contexto da pandemia.

Durante essa atividade piloto identificou-se que seria necessário uso de gravação por duas pessoas (pesquisador e observador) para que pudesse garantir a gravação, caso houvesse interrupção da chamada. Foram necessários ajustes na estrutura de duas questões norteadoras para melhor direcionamento durante a dinâmica do grupo online. Também foi pactuado previamente com o observador que sinalizasse ao pesquisador qualquer movimento, dúvidas no chat ou interrupção não percebida pelo mesmo.

Para a realização dos GF online, construiu-se um roteiro norteador da coleta de dados com três momentos: (a) apresentação dos participantes, uma breve explicação sobre a proposta da pesquisa, esclarecimentos sobre as dúvidas e confirmação do envio do TCLE online; (b) perguntas disparadoras focalizando as perspectivas dos residentes a acerca do problema da atuação e formação no contexto de pandemia; (c) finalização com oportunidade para alguma colocação final que possa não ter sido mencionada anteriormente e encerrava-se com agradecimentos finais aos participantes.

Nos três encontros com grupos diferentes, à medida que os residentes expressavam seus sentimentos, falas e concepções ficava evidente que as argumentações e desenvolvimento das discussões aconteciam naturalmente. Foi possível constatar a promoção de debate de forma criativa, alegre e sem desaprovações ou críticas. 
Entretanto, no decorrer da realização dos grupos focais na modalidade online, foi possível observar algumas potencialidades e desafios que serão descritas no quadro 1. Por ser um ambiente virtual, o mesmo apresenta suas próprias particularidades, ou seja, neste contexto, houve favorecimento de alguns questões, como: tempo de fala dos participantes permitindo aos mesmos realizar suas reflexões sem interrupções, obstáculo comum nos grupos focais presenciais, outra questão de bastante evidência foi a interação e envolvimento do grupo quando um dos participantes tinha o sinal de internet interrompido, o grupo se mobiliza em prol do retorno do participante fato que corrobora a aproximação do grupo com uso deste tipo de tecnologia.

A experiência prévia dos participantes com reuniões online desde o início da pandemia também favoreceu o contexto da coleta de dados, uma vez que as habilidades para essa forma de participação, requer alguma prática quanto ao processo de comunicação, com percepção e favorecimento das falas de todos, sem sobreposição de falas. Todos conseguiram se expressar de forma clara, com encadeamento de ideias entre os participantes, tendo facilidade em se expor e apresentar os conteúdos abordados, proporcionando reflexões sobre os disparadores sem muitas interferências do coordenador, de forma que não houve momentos de silêncio ou dificuldades de expor as ideias, tendo respeito pela diversidade de opiniões.

Por outro lado, com relação aos desafios, ficou evidente a dificuldade do pesquisador em atentar-se a todos os movimentos que ocorriam durante os encontros, as comunicações verbais e não verbais ficaram mais difíceis de serem percebidas.

Todos esses processos apontados de potencialidades e desafios podem ser melhor explorados e vivenciados como possibilidades para novas pesquisas que exijam o mesmo formato, como em momentos de ocorrência de pandemias e também em perspectivas de novas formas de utilização da técnica.

Quadro 1. Potencialidades e desafios na realização do grupo focal online, 2021.

\begin{tabular}{|c|c|}
\hline Potencialidades & Desafios \\
\hline $\begin{array}{l}\text { Conveniência de } \\
\text { agendamento }\end{array}$ & $\begin{array}{l}\text { Manter a atividade sem interrupção do } \\
\text { sinal de internet para dar continuidade } \\
\text { as falas/dinâmica }\end{array}$ \\
\hline $\begin{array}{l}\text { Experiência prévia dos } \\
\text { participantes da coleta de } \\
\text { dados com atividade em grupo } \\
\text { online } \\
\text { Uso prévio de metodologias }\end{array}$ & $\begin{array}{l}\text { Observador fica limitado a posturas e } \\
\text { gestos apresentados na delimitação do } \\
\text { video }\end{array}$ \\
\hline $\begin{array}{l}\text { ativas auxiliou no } \\
\text { envolvimento e iniciativa dos } \\
\text { participantes durante a } \\
\text { atividade }\end{array}$ & $\begin{array}{l}\text { Sinal de internet ruim interrompendo e } \\
\text { atrasando o início e a participação } \\
\text { durante a atividade }\end{array}$ \\
\hline $\begin{array}{l}\text { Menor inibição para discorrer } \\
\text { sobre a temática }\end{array}$ & $\begin{array}{l}\text { Solicitação de que camera do } \\
\text { computador dos participantes sempre } \\
\text { ficasse aberta }\end{array}$ \\
\hline
\end{tabular}

Fonte: dados da pesquisa.

\section{Reflexões sobre a Estratégia de Coleta de Dados}

Com o novo cenário imposto pela pandemia, os residentes da SC e SM que estavam inseridos na rede de atenção primária e secundária do município de Marília/SP, foram realocados para outros serviços, evitando o contato direto com os pacientes suspeitos e confirmados pela COVID-19. Por conta dessas medidas e, principalmente, pelo uso do isolamento social, fez com que a coleta de dados das pesquisas qualitativas, por meio de grupo focal presencial, fosse conduizidas para plataformas digitais como uma forma de diminuir riscos de contato e transmissão do vírus. 
A técnia de grupo focal surge como uma aborgadem qualitativa de coleta de dados e uma estratégia de ponte para a pesquisa científica e o conhecimento local, sendo amplamente usada na pesquisa de conversação.

O método, portanto, tem maior popularidade surgindo da pesquisa participativa nas ciências sociais acadêmicas durante a década de 1980 (Nyumba, Wilson, Derrick, \& Mukherjee, 2018).

Ainda neste sentido, a discussão do grupo focal consiste em quatro etapas principais, as quais incluem desenho de pesquisa, coleta de dados, análise e relato de resultados (Nyumba et al., 2018). Assim, o processo começa com a identificação do objetivo e a definição dos objetivos-chave de pesquisa do estudo, identificação dos participantes, composição do grupo, a qual dependerá do objetivo principal da pesquisa, elaboração das questões norteadoras compostas de perguntas abertas destinadas a capturar as experiências em profundidade dos entrevistados, produção de dados qualitativos e observacionais e os resultados o pesquisador precisa consolidar em um relatório coerente para divulgação (Rosenthal, 2016).

Em decorrência da dinâmica e configuração dos programas de residências e do cenário atual de pandemia, realizou-se apenas um encontro por categoria (R1 e R2) e programa, pois independentemente do número de reuniões o importante é considerar o tempo de duração, sendo assim, nossos encontros tiveram duração em médica de uma hora e meia, evitando possível fadiga por parte dos participantes.

Inicialmente, pensou-se em agrupar os residentes do primeiro e segundo ano de atividades. No entanto, percebeu-se que um número maior que oito participantes no formato online interfere na qualidade do conteúdo apresentado pelos integrantes do grupo, aumentando o tempo de realização da atividade e número de oportunidades para cada um poder se sentir à vontade em participar sem interromper o outro e abordar todos os aspectos propostos no roteiro da atividade. Com isso, a partir do primeiro encontro, com os R2 do programa RIMS Saúde Coletiva e Saúde Mental, optou-se por separar em dois grupos, sendo um composto por R1 da Saúde Coletiva e outro por R1 da Saúde Mental, para a coleta de dados, o que proporcionou maior participação e profundidade dos conteúdos.

Todos os encontros foram mediados pelo pesquisador e um observador. Assim, durante os encontros o pesquisador assume um papel de "facilitador" ou de "moderador" no decorrer das discussões, ao contrário de uma entrevista que o pesquisador adota um papel mais periférico, em vez de central, como em uma discussão de grupo focal (Rosenthal, 2016).

No caso do coordenador, mas também do observador, a experiência prévia com trabalho em grupo mostra-se importante, uma vez que a condução da atividade pode conter desafios para que os participantes, caso não tenham experiência de trabalho em grupo e uso da ferramenta online, fique prejudicada ou dificultada pelas interferências durante o desenvolvimento da atividade.

Com relação ao observador o mesmo tem papel de captar as interações não verbais e o impacto da dinâmica do grupo e documentar o conteúdo geral da discussão, complementando assim os dados (Rosenthal, 2016). Neste aspecto, a coleta de dados durante a discussão pode ser documentada pela gravação de áudio, bem como o observador conseguiu realizar anotações sobre as atitudes dos participantes, mesmo sendo online, tendo aspectos das reações deles que vão auxiliar na análise dos dados.

No entanto, cada método apresenta diferentes vantagens e desvantagens, nas quais os pesquisadores devem considerar questões específicas do contexto ao selecionar um método de coleta de dados (Rosenthal, 2016). Uma das hipóteses para o bom andamento da coleta de dados com os residentes foi que os programas de residência trabalham com metodologias ativas e isso já proporciona o protagonismo dos participantes, sustentando os objetivos da pesquisa em questão. 
A abordagem da formação profissional com métodos ativos promove um estudante proativo, responsável e independente, além do desenvolvimento dos aspectos atitudinais/afetivos. A superação das fragilidades também são trabalhadas por meio dos movimentos do processo ensino-aprendizagem.

O método ativo de ensino-aprendizagem promove a constituição da prática reflexiva e constituição do pensamento crítico acerca do que o estudante vivencia no cotidiano dos cenários de prática professional (Sobral \& Campos, 2012).

Dessa forma, foi percebido que os participantes conseguiram também ter um desempenho proativo e crítico durante a atividade de coleta de dados, podendo ser devido ao acúmulo dessas capacidades ao longo de sua formação, o que pode ter favorecido os movimentos propostos na estratégia de GF online, uma vez que a pouca habilidade no trabalho em grupo poderia ser um limitante frente a todo o contexto da atividade online. Essa poderia ser uma situação a ser melhor investigada com profundidade para futuras abordagens.

\section{Conclusões}

Conclui-se, portanto, que a realização do grupo focal na modalidade online pode sim ser considerada como uma ferramenta alternativa em cenários de pandemia como os vivenciados atualmente, que resultam na impossibilidade ou redução de se realizar reuniões presenciais, provocando aglomerações, que não são recomendadas pelos protocolos de prevenção e redução da transmissão do virus.

A realização de GF online proporciona uma continuidade das pesquisas qualitativas já em andamento ou em futuros projetos, evitando maiores impactos em seus desenvolvimentos até o retorno das atividades presenciais em condições mais seguras.

Entretanto, devemos considerar que o ambiente virtual tem suas particularidades, como imprevisto com a rede de internet acessada, dificuldades de grupos especificos com esse tipo de tecnologia e dificuldade por parte do pesquisador e observador com a habilidade do uso das ferramentas digitais nas plataformas, necessitando de formação anterior ao início das atividades de coleta de dados.

Outro aspecto a ser observado é o acúmulo de experiência do coordenador e observador com o trabalho em grupo, o que já pode ter interferencia no GF presencial, potencializando os problemas no formato online na condução e na leitura corporal e em todos os movimentos ocorridos durante a dinâmica grupal.

Neste sentido, a composição do grupo focal e uso deste tipo de método deve ser cuidadosamente considerada pelos pesquisadores, pois pode ou não favorecer o andamento da coleta de dados. Contudo, nossa experiência neste aspecto foi bastante produtiva justificada posssivelmente pela aproximção dos residentes como a abordagem em grupo.

Assim, inclui-se mais uma possibilidade de coleta de dados para a realização de pesquisas qualitativas não presenciais, todavia devemos considerar a necessidade de novos e futuros estudos nesta modalidade para compor maiores afirmações e considerações.

\section{Referências}

Barbosa Júnior, L. B., Gama, I. C. S., de Oliveira, B. F., Pessalacia, J. D. R., Martins, T. C. R., \& Santos, E. M. (2020). Uso de tecnologias na formação interprofissional de acadêmicos de Medicina durante a pandemia de COVID-19. Research, Society and Development, 9(11), e2179119594.

Busanello, J., Lunardi Filho, W. D., da Costa Kerber, N. P., Santos, S. S. C., Lunardi, V. L., \& Pohlmann, F. C. (2013). Grupo focal como técnica de coleta de dados. Cogitare Enfermagem, 18(2), 358-364. 
Hammerschmidt, K. S. A., Bonatelli, L. C. S., \& Carvalho, A. A. (2020). Caminho da esperança nas relações envolvendo os idosos: olhar da complexidade sobre pandemia da COVID-19. Texto \& Contexto-Enfermagem, 29. e20200132.

Kienle, G. S., \& Kiene, H. (2011). Como escrever um relato de caso. Arte Med Ampl, 31(2), 34-37.

Lucena, E. H. G., Freire, A. R., Freire, D. E. W. G., Araújo, E. C. F., Lira, G. N., Brito, A. C. M., Padilha, W. W. N., \& Cavalcanti, Y. W. (2020). Offer and Use of Oral Health in Primary Care Before and After the Beginning of the COVID-19 Pandemic in Brazil. Pesquisa Brasileira em Odontopediatria e Clínica Integrada, 20(Supl. 1), e0139.

Minayo, M. C. S., Deslandes, S. F., \& Gomes, R. (2011). Pesquisa social: teoria, método e criatividade: Editora Vozes Limitada.

Mueller, V. (2018). O papel das Residências em Saúde na qualificação e expansão da Atenção Primária: saberes e impressões na perspectiva do usuário. (dissertação mestrado). Universidade do Estado do Rio de Janeiro. Disponível em: http://152.92.4.120:8080/browse?type=author\&value=Mueller\%2C+Vanessa

Nyumba, T. O., Wilson, K., Derrick, C. J., \& Mukherjee, N. (2018). The use of focus group discussion methodology: Insights from two decades of application in conservation. Methods in Ecology and evolution, 9(1), 20-32.

Oliveira, A. C., Lucas, T. C., \& lquiapaza, R. A. (2020). O que a pandemia da covid-19 tem nos ensinado sobre adoção de medidas de precaução? Texto \& Contexto-Enfermagem, 29, e20200106.

Quintella, C. M., Mata, A. M. T., Ghesti, G. F., \& Tavares, P. M. d. A. L. (2020). Vacinas para Coronavírus (COVID-19; SARS-COV-2): mapeamento preliminar de artigos, patentes, testes clínicos e mercado. Cadernos de Prospecção, 13(1), 3.

Ressel, L. B., Beck, C. L. C., Gualda, D. M. R., Hoffmann, I. C., Silva, R. M., \& Sehnem, G. D. (2008). O uso do grupo focal em pesquisa qualitativa. Texto \& Contexto-Enfermagem, 17(4), 779-786.

Rocha, E. N., \& Lucena, A. F. (2018). Projeto Terapêutico Singular e Processo de Enfermagem em uma perspectiva de cuidado interdisciplinar. Revista Gaúcha de Enfermagem, 39, e20170057.

Rodrigues, N. H., \& Silva, L. G. A. (2020). Gestão da pandemia coronavírus em um hospital: relato de experiência profissional. Journal of Nursing and Health, 10(n. esp.), e20104004.

Rosenthal, M. (2016). Qualitative research methods: Why, when, and how to conduct interviews and focus groups in pharmacy research. Currents in pharmacy teaching and learning, 8(4), 509-516.

Schmidt, B., Crepaldi, M. A., Bolze, S. D. A., Neiva-Silva, L., \& Demenech, L. M. (2020). Saúde Mental e Intervenções Psicológicas Diante da Pandemia do Novo Coronavírus (COVID-19). Estudos de Psicologia (Campinas), 37, e200063.

Sobral, F. R., \& Campos, C. J. G. (2012). Utilização de metodologia ativa no ensino e assistência de enfermagem na produção nacional: revisão integrativa. Revista da Escola de Enfermagem da USP, 46(1), 208-218.

Souto, L. R. F., \& Travassos, C. (2020). Plano Nacional de Enfrentamento à Pandemia da Covid-19: construindo uma autoridade sanitária democrática. Editorial. Saúde em Debate, 44 (126), 587-589. 